\title{
Torsion Studies of Thermoelasticity and Stress-Strain Isotherms of Unimodal, Bimodal, and Filled Networks of Poly(dimethylsiloxane)
}

\author{
Jianye WeN and James E. MARK \\ Department of Chemistry and the Polymer Research Center, \\ The University of Cincinnati, Cincinnati, \\ OH 45221-0172, U.S.A.
}

(Received March 11, 1993)

\begin{abstract}
End-linking reactions were used to convert chains of poly(dimethylsiloxane) (PDMS) into (i) unfilled networks having either unimodal or bimodal chain-length distributions, or (ii) filled networks in which either a separately-prepared zeolite had been blended into the polymer prior to end linking, or silica was precipitated into the networks afterwards. The resulting elastomers were studied in torsion, the aspect of particular interest in the case of the unfilled networks being the fraction $M_{\mathrm{e}} / M$ of the total couple which is due to changes in conformational energy. The values of $M_{\mathrm{e}} / M$ were found to be independent of crosslink density, extent of torsional strain, and extent of any elongation imposed during the torsion. The values for the bimodal networks, however, were much smaller than those for the unimodal ones, presumably because of the non-Gaussian nature of the short chains. None of the unimodal or bimodal unfilled networks showed any upturn in modulus at high torsional deformations similar to the upturns shown by bimodal networks in elongation, biaxial extension, and shear. Neither did the filled networks, but they did show significant increases in modulus in general, thus demonstrating reinforcement at least in this regard.
\end{abstract}

KEY WORDS Torsion / Poly(dimethylsiloxane) Elastomers / Bimodal

Networks / Thermoelasticity / Energetic Contributions to the Elastic

Response / Stress-Strain Isotherms / Zeolites / In-Situ Precipitated Fillers /

Reinforcement /

Most experimental studies of the elastomeric properties of polymer networks employ uniaxial elongation or equilibrium swelling as the type of deformation primarily because such experiments are relatively easy to carry out. ${ }^{1,2}$ As a specific example, very little research has been done on networks in torsion, in spite of the fact that this deformation is important from both practical and fundamental points of view. With regard to the former, it is sufficient to note that many elastomers are used in torsion, or in torsion combined with elongation. The importance with regard to fundamentals has to do with the simplicity of the relationships obtained with regard to the dependence of the force on temperature and on extent of de- formation. Specifically, the correction term for the desired conversion of the forcetemperature ("thermoelastic") coefficient from constant pressure to constant volume is not dependent on extent of deformation, as it is in elongation measurements. In addition, the stress-strain relationship in torsion is found to be much closer to the form predicted by the simplest molecular theories ${ }^{1,2}$ than is the case for elastomers in extension. ${ }^{3-5}$

The present study of an elastomer under torsion focuses on poly(dimethylsiloxane) (PDMS), of repeat unit $\left[-\mathrm{Si}\left(\mathrm{CH}_{3}\right)_{2}-\mathrm{O}-\right]$. It is an important commercial material, and it has been studied extensively (both unfilled and filled), in elongation and in compression..$^{2,7-12}$ 
Of particular interest is the fact that PDMS networks having a bimodal distribution of network chain lengths showed unusual thermoelastic behavior in elongation. The present investigation was therefore undertaken to explore the thermoelastic and stress-strain behavior of unimodal, bimodal, and filled PDMS elastomers in torsion.

\section{EXPERIMENTAL}

\section{Materials}

The polymers employed, three hydroxylterminated samples of PDMS with quoted number-average molecular weights $M_{n}$ of 500, 18000 , and $26000 \mathrm{~g} \mathrm{~mol}^{-1}$, respectively, were obtained from the Huls Americal Company. Values of $M_{w}, M_{n}$, and $M_{w} / M_{n}$ were independently determined using a Waters 746 gel permeation chromatography (GPC) instrument and are presented in Table I. The end-linking molecule was tetraethoxysilane (TEOS) $\left[\mathrm{Si}\left(\mathrm{OC}_{2} \mathrm{H}_{5}\right)_{4}\right]$ and was supplied by the Petrach Systems company, and the catalyst for the reaction, stannous octoate, was obtained from the Pfaltz and Bauer Company. The zeolite filler employed in some cases was a sodium aluminosilicate (Zeolite 13X), and was obtained from Amresco, Inc. The material chosen for the in-situ hydrolyses to silica was also the TEOS.

\section{Preparation of Networks}

Hydroxyl-terminated PDMS chains were end linked with TEOS in the usual man-

Table I. Molecular weights of PDMS chains

\begin{tabular}{|c|c|c|c|c|}
\hline \multirow{3}{*}{$\begin{array}{c}\text { Petrach } \\
\text { designation }\end{array}$} & \multirow{3}{*}{$\frac{\text { Quoted } M_{n}}{\mathrm{~g} \mathrm{~mol}^{-1}}$} & \multicolumn{3}{|c|}{ GPC results } \\
\hline & & $M_{n}$ & $M_{w}$ & \\
\hline & & $\mathrm{g} \mathrm{mol}^{-1}$ & $\mathrm{~g} \mathrm{~mol}^{-1}$ & $W_{w /}$ \\
\hline PS 343 & 26000 & 22100 & 34600 & 1.57 \\
\hline PS 342.5 & 18000 & 15600 & 27900 & 1.79 \\
\hline PS 340 & 500 & 344 & 408 & 1.20 \\
\hline
\end{tabular}

ner. ${ }^{2,12,13}$ Five unfilled networks were prepared, and are described in the first five columns of Table II. As can be seen, two had a unimodal distribution of network chain lengths, and three a bimodal distribution. Each mixture of weighed amounts of PDMS, TEOS, and catalyst (and zeolite filler in some cases) was poured into a cylindrical mold made of Teflon ${ }^{\circledR}$. The mold was then placed under vacuum for several minutes to remove air bubbles, after which the cross-linking reaction was allowed to proceed at room temperature for three days. The cylindrical samples thus prepared were then removed from the molds and weighed. Each network was extracted with toluene and methanol, dried, and reweighed. The weights before and after the extractions were used to calculate values of the sol fraction, consisting of polymer chains not incorporated in the network structure.

For the networks to be filled with in-situ generated silica, the extracted samples were weighed and then immersed in a solution of ethanol containing TEOS until the desired amount of TEOS was absorbed. The swollen, samples were then placed into a $2 \mathrm{wt} \%$ aqueous solution of diethyl amine, and the hydrolysis and condensation to $\mathrm{SiO}_{2}$ permitted to occur at room temperature for the desired period of time. After the reaction, each sample was dried and reweighed, and the difference in weights before and after the precipitation was used to calculate the amount of filler introduced.

Table II. Sample compositions and sol fractions

\begin{tabular}{|c|c|c|c|c|c|}
\hline \multirow[t]{2}{*}{ Sample } & \multicolumn{2}{|c|}{$\frac{M_{n}}{\mathrm{~g} \mathrm{~mol}^{-1}}$} & \multicolumn{2}{|c|}{$\mathrm{Mol} \%$} & \multirow{2}{*}{$\begin{array}{c}\begin{array}{c}\text { Sol } \\
\text { fraction }\end{array} \\
w t \%\end{array}$} \\
\hline & $\begin{array}{l}\text { Long } \\
\text { chains }\end{array}$ & $\begin{array}{l}\text { Short } \\
\text { chains }\end{array}$ & $\begin{array}{l}\text { Long } \\
\text { chains }\end{array}$ & $\begin{array}{l}\text { Short } \\
\text { chains }\end{array}$ & \\
\hline U-1 & 18000 & & 100 & & 4.9 \\
\hline U-2 & 26000 & & 100 & & 6.5 \\
\hline B-1 & 26000 & 500 & 10 & 90 & 4.3 \\
\hline B-2 & 26000 & 500 & 6 & 94 & 4.1 \\
\hline B-3 & 18000 & 500 & 10 & 90 & 3.9 \\
\hline
\end{tabular}




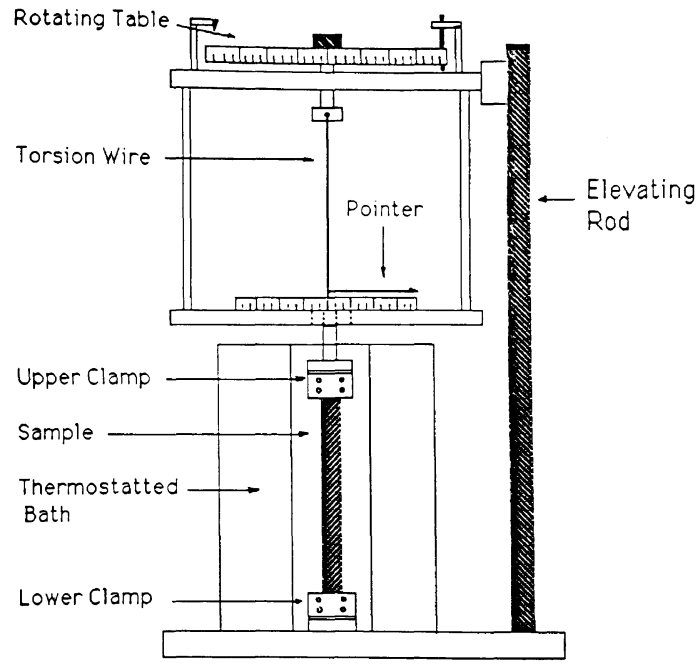

Figure 1. Schematic representation of the torsion apparatus.

\section{Apparatus}

The home-built torsion apparatus employed was similar to that designed by Gent and Kuan, ${ }^{5}$ and is shown schematically in Figure 1. The cylindrical sample, about $35 \mathrm{~mm}$ long and $3.5 \mathrm{~mm}$ in diameter, was secured between two specially-designed clamps, and was surrounded by a long thin glass tube which was immersed in a thermostatted water bath to achieve good temperature control. Both the upper and lower clamps could be moved so as to impose a desired elongation on the sample, as well as the desired torsion. The upper clamp was attached to the end of a fine torsion wire, the upper end of which was attached to a rotating table calibrated in degrees. A pointer was also attached to the upper clamp, and the extent of its rotation above an aluminum disc was used to establish the initial degree of twist imposed on the sample, in its reference state.

\section{Mechanical Property Measurements}

Any change of the couple $M$ arising from a change of temperature or other variable could be measured by the twist of the torsion wire required to return the pointer to its reference position. Thus, the couple-temperature (stress-temperature) relations could be determined for a constant amount of twist (superimposed upon a constant extension if necessary) in the temperature range of $20-95^{\circ} \mathrm{C}$. The following specific steps were employed: a given amount of twist was imposed on the sample by rotating the upper clamp via the rotating table. Couple readings were taken at 10 to $15^{\circ} \mathrm{C}$ intervals after sufficient time (around $20 \mathrm{~min}$ ) had been allowed for the attainment of thermal equilibrium at each temperature. Measurements were taken in the order of decreasing temperature, with additional points subsequently taken with increasing tempemperature to test for reversibility. Between the chosen temperatures, the sample was heated or cooled at the rate of $1-2^{\circ} \mathrm{C}$ per minute.

Couple-strain isotherms were obtained using a sequence of increasing values of twist. Couple readings were taken after sufficient time had been allowed for the attainment of stress equilibrium ( $20 \mathrm{~min}$ for unfilled networks and $12 \mathrm{~h}$ for filled networks). Measurements were carried out to the rupture points of the samples. The absolute values of the couple were obtained from the torsion constant of the torsion wire, which was determined by a standard ASTM method.

\section{THEORY}

\section{Stress-Temperature Relationships}

Although the elastomeric force is primarily due to the entropy decreases accompanying the stretching out of network chains, there can also be an energetic component resulting from the fact that different spatial configurations generally correspond to different conformational energies. ${ }^{14}$ Even though the energetic contribution $f_{\mathrm{e}}$ to the total retractive force $f$ is generally small, it can be measured from the temperature dependence of retractive force in a sample held at a fixed deformation. ${ }^{1,2}$ In his theoretical analysis of Gaussian networks in elongation, Flory derived equations which 
relate the energetic component $f_{\mathrm{e}}$ of the tensile force $f$ to the stress-temperature coefficient. In the condition of constant volume which is required by the thermodynamics, the result is $^{1,2,14-16}$

$$
\frac{f_{\mathrm{e}}}{f}=1-(T / f)(\partial f / \partial T)_{\mathrm{L}, \mathrm{v}}
$$

Under conditions of constant pressure, which are experimentally more convenient, the expression is

$$
\frac{f_{\mathrm{e}}}{f}=1-(T / f)(\partial f / \partial T)_{\mathrm{L}, \mathrm{P}}-\beta T /\left(\alpha^{3}-1\right)
$$

where $\beta$ is the volume expansion coefficient, $\alpha$ is the extension ratio, and the last term represents the Gaussian correction for the conversion.

The corresponding analysis for the case of torsion has been given by Treloar. ${ }^{3}$ The relative energetic contribution $M_{\mathrm{e}}$ to the torsion couple $M$ can be obtained from the equation

$$
\frac{M_{\mathrm{e}}}{M}=1-(T / M)(\partial M / \partial T)_{\mathrm{L}, \mathrm{P}, \psi}+\beta T
$$

where $M$ is the couple required to maintain a twist of a specified number of radians per unit stretched length. It should be noted that the Gaussian conversion term $\beta T$ in this equation does not depend on the extent of the deformation. In any case, estimates of $M_{\mathrm{e}} / M$ and $f_{\mathrm{e}} / f$ for (unfilled) networks of the same polymer should be identical.

The additional equation

$$
\mathrm{d} \ln \left\langle r^{2}\right\rangle_{0} / \mathrm{d} T=(1 / T)\left(M_{\mathrm{e}} / M\right)
$$

is an important bridge equation in that it expresses the thermodynamic ratio $M_{\mathrm{e}} / M$ in terms of a molecular quantity, the temperature coefficient of the unperturbed dimensions of the network chains. ${ }^{1,2,14}$ It also connects the former quantity, obtainable only from elasticity measurements, with the latter quantity, which can also be determined from viscosity-temperature measurements on dilute polymer solutions.

\section{Stress-Deformation Relationships}

The shear modulus $G$ can calculated from the torsion strain $\psi a$ by use of the equation ${ }^{1}$

$$
G=2 M / \pi \psi a^{4}
$$

where $M$ is the torsion couple, $\psi$ is the torsion (expressed in radians per unit length of the strained axis), and $a$ is the unstrained radius. According to Mooney's equation, the simple shear stress for the torsion of a cylinder may be written ${ }^{1}$

$$
t_{\mathrm{qz}}=2 \psi a\left(C_{1}+C_{2}\right)
$$

where $C_{1}$ and $C_{2}$ are elastic constants. Since the modulus $G$ is equal to $t_{\mathrm{qz}} / \psi a$, it should be independent of deformation.

\section{RESULTS AND DISCUSSION}

\section{Sol Fractions}

The values of the sol fraction of polymer obtained from the extraction measurements are given in the final column of Table II. They are seen to be relatively small, and indicate extents of end linking sufficiently large for the purposes at hand.

\section{Thermoelastic Studies}

Typical couple-temperature plots for unimodal and bimodal networks are shown

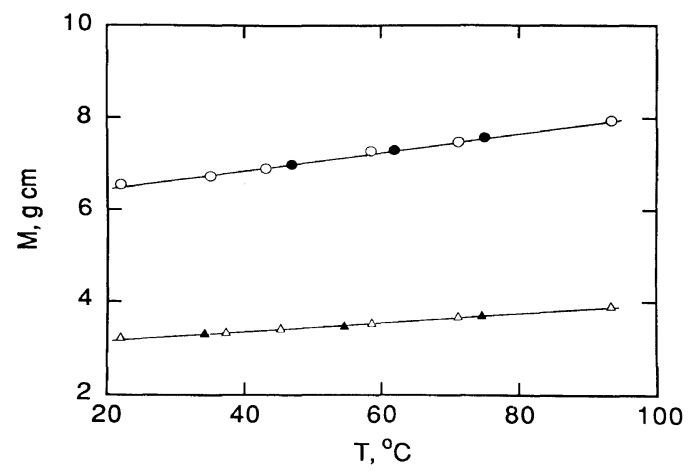

Figure 2. Couple-temperature plots for the unimodal PDMS networks, U-1 $(O)$ and $U-2(\triangle)$. The filled symbols locate data obtained to check for reversibility. 


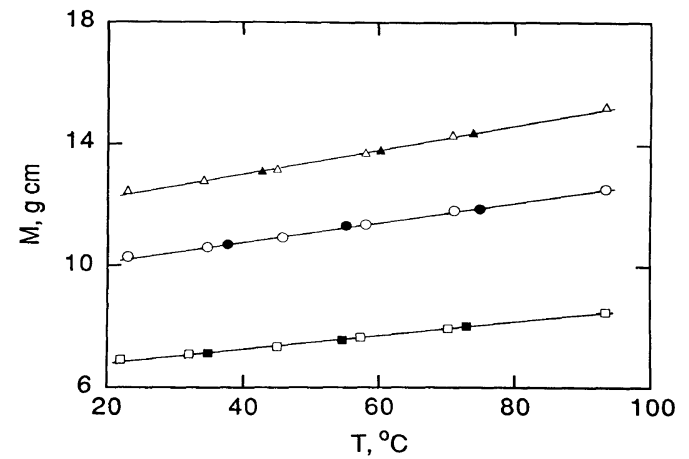

Figure 3. Couple-temperature plots for the bimodal networks, B-1 $(\square)$, B-2 $(\triangle)$, and B-3 $(\bigcirc)$. The filled symbols located data obtained to check for reversibility.

Table III. Values of $M_{\mathrm{e}} / M$ at different torsional strains

\begin{tabular}{lll} 
& $\psi a$ & $M_{\mathrm{e}} / M$ \\
\hline $\mathrm{U}-1$ & 0.25 & 0.12 \\
& 0.30 & 0.13 \\
$\mathrm{U}-2$ & 0.316 & 0.13 \\
& 0.493 & 0.13 \\
& 0.663 & 0.13 \\
$\mathrm{~B}-1$ & 0.248 & 0.07 \\
& 0.328 & 0.08 \\
$\mathrm{~B}-2$ & 0.203 & 0.07 \\
& 0.312 & 0.06 \\
$\mathrm{~B}-3$ & 0.224 & 0.06 \\
& 0.315 & 0.07 \\
\hline
\end{tabular}

in Figures 2 and 3, respectively. In all cases a reversible, linear relationship was found to exist between the torsion couple and temperature. The couple increases with increase in temperature, since the primary effect is an increase in the chaotic chain motions which drive the system in the direction of maximum randomness (which is the undeformed state). ${ }^{1,2}$

The values of $M_{\mathrm{e}} / M$ for unimodal and bimodal networks for different values of the torsional strain, $\psi a$, are given in Table 3 . The results are seen not to depend either on torsional strain or on the differences in cross-link density between U-1 and U-2, and among B-1, B-2, and B-3. The values obtained at different extension ratios are given in Table IV, and are also seen to be independent of the magnitude of this strain, and the cross-link
Table IV. Values of $M_{\mathrm{e}} M$ at different extension ratios

\begin{tabular}{ccc}
\hline & $\alpha$ & $M_{\mathrm{e}} / M$ \\
\hline $\mathrm{U}-2$ & 1.01 & 0.13 \\
& 1.12 & 0.13 \\
& 1.22 & 0.11 \\
$\mathrm{~B}-1$ & 1.41 & 0.11 \\
& 1.01 & 0.07 \\
$\mathrm{~B}-2$ & 1.28 & 0.05 \\
& 1.01 & 0.06 \\
& 1.14 & 0.07 \\
\hline
\end{tabular}

density. These results thus support the basic postulate of rubber elasticity theory that although intermolecular interactions unquestionably occur in rubberlike materials, these interactions are independent of the configurations of the network chains and, therefore, are also independent of the extent of deformation of the network structure. ${ }^{2}$

All the values of $M_{\mathrm{e}} / M$ average to 0.12 for the unimodal networks, and to 0.06 for the bimodal ones. This is remarkably similar to the results obtained on PDMS elastomers in elongation, in which unimodal networks gave an average value of $f_{\mathrm{e}} / f$ of 0.20 , while bimodal ones gave $0.08 .{ }^{17}$ The difference is presumably due to the fact that the short chains are very non-Gaussian. The fact that the bimodal networks give smaller values of these ratios suggests that the non-Gaussian effect simply involves the chains having very few spatial arrangements at this chain length. If bondangle deformation or bond stretching were involved, these ratios might be expected to be larger since these two modes of deformation require large energy increases. This conclusion is consistent with a recent spectroscopic study that found little evidence for these modes of deformation in bimodal PDMS networks stretched to their rupture points. ${ }^{18}$

Equation 4 permits conversion of the thermodynamic quantity $M_{\mathrm{e}} / M$ to the temperature coefficient $10^{3} \mathrm{~d} \ln \left\langle r^{2}\right\rangle_{0} / \mathrm{d} T$ of the unperturbed dimensions of the network chains. The value thus obtained, 0.45 , is in good 
agreement with the value, 0.59 , obtained from viscosity-temperature measurements on uncrosslinked PDMS chains. ${ }^{14}$ It arises from the fact that increase in temperature increases the number of higher-energy gauche conformations, and these correspond to relatively high spatial extension in the case of the PDMS chain.

\section{Stress-Deformation Relationships}

Figure 4 shows the dependence of the couple $M$ on the elongation or extension ratio imposed at a constant torsion. The observed decrease in the couple with increase in elongation is presumably analogous to the decrease in modulus with deformation in simple elonga-

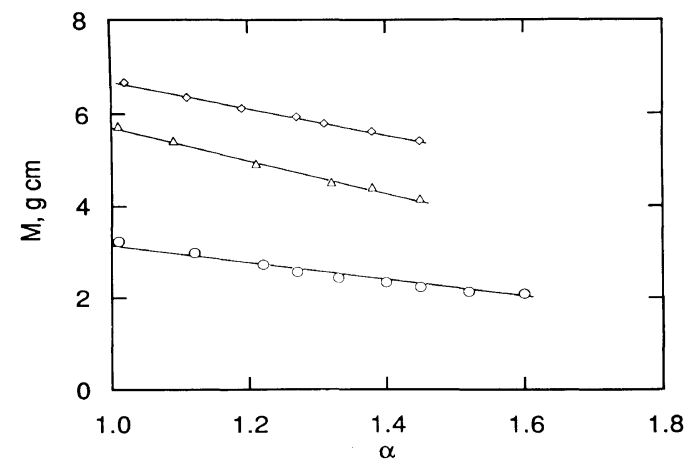

Figure 4. Couple-elongation relationships at $25^{\circ} \mathrm{C}$ for unimodal and bimodal networks, at fixed torsional strains of 0.250 for $\mathrm{U}-1(\triangle), 0.316$ for $\mathrm{U}-2(\bigcirc)$, and 0.248 for B-1 $(\diamond)$.

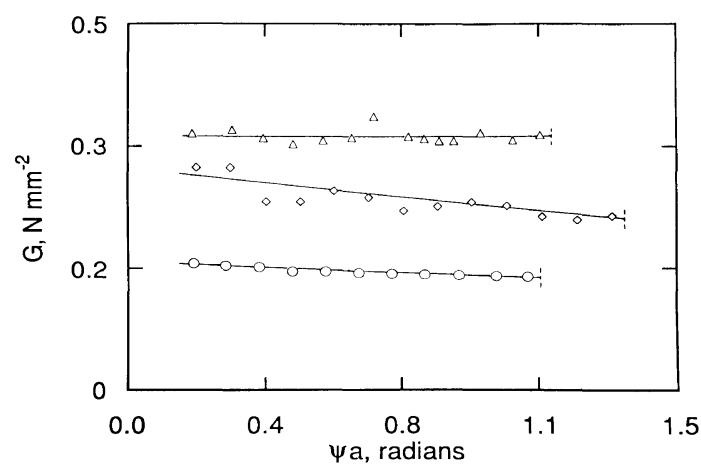

Figure 5. Dependence of the couple on torsional strain for the unimodal and bimodal networks at $25^{\circ} \mathrm{C}$ : U-1 (O), B-1 $(\triangle)$, and B-2 $(\diamond)$. tion, represented by the Mooney-Rivlin $2 \mathrm{C}_{2}$ constant ${ }^{1,2}$ and ascribed to the deformation becoming increasingly nonaffine as the elongation increases. ${ }^{2,19-21}$

The couple-torsional strain isotherms obtained for unfilled unimodal and bimodal PDMS networks, represented in terms of the shear modulus $G$ and torsion strain $\psi a$, are shown in Figure 5. The modulus is seen to show little or no dependence on torsional strain, in agreement with previous results. ${ }^{3-5}$ Unlike the corresponding isotherms in elongation, ${ }^{2}$ in biaxial extension, ${ }^{22}$ and in shear, ${ }^{23}$ however, there are no upturns in modulus at high deformations. Thus, in this case, the higher values of the modulus of the bimodal networks can be simply accounted for in terms of a lower

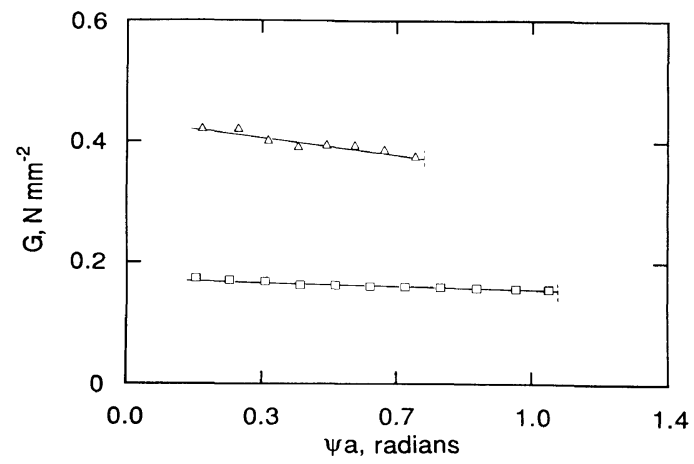

Figure 6. Couple-torsion strain plots at $25^{\circ} \mathrm{C}$ for unfilled network U-1 $(\square)$, and for one filled with zeolite $(\triangle)$.

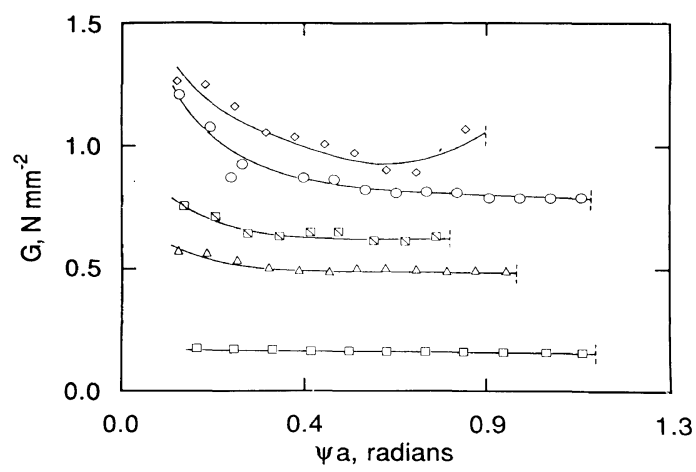

Figure 7. Couple-torsion strain plots at $25^{\circ} \mathrm{C}$ for PDMS U-1 networks filled with in-situ precipitated silica. Values of the wt\% filler were: $0.0(\square), 15.5(\triangle), 17.3(\triangle), 19.8$ $(\bigcirc)$, and $26.4(\diamond)$. 
average value of the molecular weight between cross links. The absence of any upturns in modulus could be due either to the relative insensibility of shear modulus to torsional deformations, or simply to difficulties in reaching sufficiently high deformations.

The corresponding isotherms for the filled PDMS networks are shown in Figures 6 and 7. Again, there is little evidence for the strong upturns in modulus at high deformations observed for filled PDMS elastomers in elongation, ${ }^{2,24,25}$ in biaxial extension, ${ }^{26}$ and in shear. ${ }^{26}$ Both the zeolite filler and the in-situ generated silica fillers give substantial increases in modulus, however, indicating good reinforcement in this respect. Of the two types, the in-situ precipitated silica seems to give much better reinforcement.

It is interesting to note that the moduli of the elastomers containing large amounts of silica do decrease significantly with increase in torsional strain, and the larger the amount of filler, the bigger the decrease. This may be due to stress-induced rearrangements of the chains in the vicinity of the filler particles, but this is one of the least-understood areas of rubberlike elasticity and will require a great deal more experimental and theoretical work before definitive conclusions can be drawn.

Acknowledgments. It is a pleasure to acknowledge the financial support provided by the National Science Foundation through Grant DMR 89-18002 (Polymers Program, Division of Materials Research). The authors would also like to thank Dr. Russell A. Livigni of GenCorp for a generous donation for the torsion apparatus, and Mr. W. L. Brauntz of the Chemistry Department of The University of Cincinnati for his assistance in constructing it.

\section{REFERENCES}

1. L. R. G. Treloar, "The Physics of Rubber Elasticity," Oxford University Press, Clarendon, 1975.

2. J. E. Mark and B. Erman, "Rubberlike Elasticity. A Molecular Primer," Wiley-Interscience, New York, N.Y., 1988.

3. L. R. G. Treloar, Polymer, 10, 291 (1969).

4. P. H. Boyce and L. R. G. Treloar, Polymer, 11, 21 (1970).

5. A. N. Gent and T. H. Kuan, J. Polym. Sci., Polym. Phys. Ed., 11, 1723 (1973).

6. G. Martin and F. S. Kipping, J. Chem. Soc., 95, 302 (1909).

7. A. J. Barry and H. N. Beck, "Inorganic Polymers," Academic Press, New York, N.Y., 1962, p 189.

8. K. A. Andrianov, in "Metalorganic Polymers," Vol. I, Wiley-Interscience, New York, N.Y., 1965, p 48.

9. J. R. Hamilton, "Silicone Technology," Interscience, New York, N.Y., 1970.

10. J. E. Mark, Rubber Chem. Technol., 46, 593 (1973).

11. J. E. Mark, J. Polym. Sci., Macromol. Rev., 11, 135 (1976).

12. J. E. Mark and J. L. Sullivan, J. Chem. Phys., 11, 1006 (1977).

13. M. A. Llorente, A. L. Andrady, and J. E. Mark, J. Polym. Sci., Polym. Phys. Ed., 19, 621 (1981).

14. P. J. Flory, "Statistical Mechnics of Chain Molecules," Interscience, New York, N.Y., 1969.

15. P. J. Flory, A. Ciferri, and C. A. J. Hoeve, J. Polym. Sci., 45, 235 (1960).

16. A. Ciferri, C. A. J. Hoeve, and P. J. Flory, J. Am. Chem. Soc., 83, 1015 (1961).

17. Z. M. Zhang and J. E. Mark, J. Polym. Sci., Polym. Phys. Ed., 20, 473 (1982).

18. L. K. Silva, J. E. Mark, and F. J. Boerio, Makromol. Chem., 192, 499 (1991).

19. P. J. Flory, J. Chem. Phys., 66, 5726 (1977).

20. P. J. Flory and B. Erman, Macromolecules, 15, 800 (1982).

21. P. J. Flory and B. Erman, J. Polym. Sci., Polym. Phys. Ed., 22, 49 (1984).

22. P. Xu and J. E. Mark, J. Polym. Sci., Polym. Phys. Ed., 29, 355 (1991).

23. S. Wang and J. E. Mark, J. Polym. Sci., Polym. Phys. Ed., 30, 801 (1992).

24. A. M. S. Al-ghamdi and J. E. Mark, Polym. Bull., 20, 537 (1988).

25. J. Wen and J. E. Mark, J. Mater. Sci., in press.

26. S. Wang, P. Xu, and J. E. Mark, Rubber Chem. Technol., 64, 746 (1991). 